\title{
L'éducation de base au Cambodge : un système en reconstruction
}

Erick Garnier et Gérard Renou

\section{OpenEdition}

1 Journals

Édition électronique

URL : https://journals.openedition.org/ries/1975

DOI : 10.4000/ries. 1975

ISSN : 2261-4265

Éditeur

France Education international

Édition imprimée

Date de publication : 1 décembre 2002

Pagination : 129-138

ISBN : 978-2854-2-0555-8

ISSN : $1254-4590$

Référence électronique

Erick Garnier et Gérard Renou, "L'éducation de base au Cambodge : un système en reconstruction », Revue internationale d'éducation de Sèvres [En ligne], 31 | décembre 2002, mis en ligne le 25 novembre 2011, consulté le 05 juillet 2021. URL : http://journals.openedition.org/ries/1975 ; DOI : https://doi.org/ 10.4000/ries. 1975

Ce document a été généré automatiquement le 5 juillet 2021.

(c) Tous droits réservés 


\title{
L'éducation de base au Cambodge : un système en reconstruction
}

\author{
Erick Garnier et Gérard Renou
}

1 Depuis les accords de paix d'octobre 1991, en dépit d'aides extérieures significatives, le décollage du Cambodge s'est heurté à un certain nombre de difficultés liées à une forte poussée démographique, à un environnement économique national post-conflictuel et sous-régional fragile ainsi qu'aux aléas de la vie politique agitée d'une démocratie naissante. Dans l'ensemble des secteurs d'activité, le Cambodge a été confronté à une problématique de développement qui a conduit délibérément vers le modernisme un pays tout entier, alors que sa population, ancrée dans la culture khmère et les traditions, vivait avec des revenus de subsistance voisins de la pauvreté.

2 Le processus de changement malgré tout engagé s'est trouvé sérieusement entravé par une série de facteurs humains qui tenaient en grande partie aux traumatismes et aux fractures d'une société profondément déstructurée par un quart de siècle de conflits armés d'une rare violence.

3 Le génocide de la majeure partie des élites intellectuelles, les troubles psychosociologiques qui touchent aujourd'hui encore les survivants, la désintégration des cellules familiales, la marginalisation culturelle et les difficultés de réintégration des élites précédemment exilées et coupées des réalités contemporaines du pays en furent les principales causes.

4 Le manque chronique de personnel compétent s'est fait sentir dans tous les secteurs d'activité. Au Cambodge, comme dans bon nombre de pays en situation postconflictuelle, les perspectives de développement demeurent largement tributaires d'une capacité à surmonter le plus rapidement possible d'énormes carences en ressources humaines.

5 La jeunesse cambodgienne est aujourd'hui encore victime d'un contexte qu'elle vit avec difficulté. Exclus prématurément de l'école par manque de performance du système éducatif (un enfant sur deux quitte l'école avant la fin du cycle primaire), de nombreux adolescents se retrouvent dans la rue, souvent analphabètes ou illettrés, attirés par la ville, sans avoir été préparés à la vie active et encore moins familiarisés à l'exercice des 
métiers urbains. Le Cambodge reste en effet un pays rural où les rizières occupent environ $20 \%$ des terres. La population des campagnes représente plus de $80 \%$ de la population totale et dans certaines zones éloignées ou défavorisées, le taux de scolarisation ne dépasse pas $30 \%$.

6 Après plus de deux décennies de conflits, le pays a connu une croissance démographique considérable. Parallèlement, l'augmentation du taux de natalité a accentué les déséquilibres dans l'accès aux terres cultivables qui ne permettent plus, aujourd'hui, de satisfaire les besoins des «familles élargies » au sens sociologique du terme. Ce phénomène classique, rencontré dans bon nombre de pays en développement, est source d'importantes conséquences socio-économiques telles que :

- la désagrégation de la cellule familiale ;

- la déstructuration de l'organisation villageoise ;

- l'engorgement des zones urbaines et péri-urbaines par des populations de jeunes sans qualification et inadaptés à la vie des villes ;

- la croissance du taux de chômage ;

- la croissance des phénomènes de délinquance juvénile ;

- l'augmentation de la précarité et de ses effets secondaires en matière de santé publique, la désorganisation de la société locale conduisant inexorablement à un processus de déstabilisation politique.

\section{Le secteur éducatif cambodgien}

\section{Principes et objectifs généraux de l'éducation}

7 La Constitution du royaume du Cambodge de 1993 adopte une politique démocratique et libérale. Le chapitre six définit le droit des citoyens à une éducation de qualité à tous les niveaux et précise le rôle de l'État dans l'établissement d'un système éducatif complet et universel sur l'ensemble du territoire. La Constitution du Cambodge prévoit ainsi dans ses articles 65, 66, 67 et 68 l'égalité d'accès à l'éducation et la gratuité de l'enseignement primaire et secondaire pour tous, assortie de l'obligation scolaire pour tout le cycle de base d'une durée de 9 ans. Ces mesures s'inscrivent dans la tendance mondiale de « l'éducation pour tous » et dans les principes généraux des accords établis à l'occasion de la Conférence internationale de Jomtien de 1990 et réaffirmés à Dakar en 2000.

8 L'adoption de cette nouvelle constitution a abouti à une réforme de l'enseignement général dont une des conséquences fut l'allongement du cycle primaire, le faisant passer de cinq à six ans. Ce changement est entré en vigueur au début de l'année scolaire 1996-1997.

9 Au-delà de ces principes généraux, l'éducation au Cambodge a pour but d'assurer aux enfants un développement harmonieux afin d'en faire «d'honnêtes citoyens dotés de bonnes connaissances et d'un esprit de solidarité, œuvrant pour la réconciliation et l'unité nationale, la justice et le respect des droits de l'homme dans un esprit de travail et de responsabilité démocratique pour le respect de la devise : Nation - Religion Roi" ".

Dès 1993, le nouveau gouvernement, issu du scrutin placé sous la responsabilité des Nations Unies, a amorcé - dans l'urgence - la reconstruction du pays sur la base du «programme national de réhabilitation et de développement du Cambodge » (PNRD). 
Depuis lors, le renouveau du Cambodge s'organise selon six axes principaux d'ordre stratégiques :

- la restructuration de l'économie avec le renforcement de l'économie de marché ;

- la remise sur pied des institutions de l'État et de l'administration publique ;

- le développement rural ;

- le développement des ressources humaines;

- la reconstruction des infrastructures physiques ;

- la croissance durable de la production.

11 Comme l'ensemble des activités et services du pays, le secteur éducatif est donc sorti sinistré des événements :

- énormes insuffisances et carences en potentiel humain ;

- caractère misérable et démobilisateur des rémunérations offertes aux enseignants ;

- manque chronique de matériels didactiques (en particulier de manuels scolaires);

- absence ou insuffisance des infrastructures scolaires ;

- absence de données fiables (ressources humaines, coût, compétences, carte scolaire...).

Doté fin 1994 d'un cadre opérationnel de politique éducative avec le «plan d'investissement 1995-2000 pour l'éducation de base » estimé à 150 millions de dollars américains, le ministère de l'Éducation, de la Jeunesse et des Sports (MEJS) a entrepris de remettre sur pied son système éducatif, sans réforme d'ensemble, mais en procédant par retouches et ajustements progressifs, avec une quadruple finalité :

- améliorer la qualité de l'enseignement aux différents niveaux;

- étendre l'accès à l'école de manière équitable ;

- renforcer la gestion et la planification du secteur de l'éducation;

- élaborer le programme de développement de la jeunesse et des sports.

Offrir à tous la possibilité d'accéder à des services convenables d'éducation et de santé est considéré comme le but à atteindre et constitue l'un des piliers de la politique gouvernementale en matière de ressources humaines. Dans cette perspective, il a été envisagé d'augmenter progressivement les dépenses d'éducation en mobilisant des ressources additionnelles sur le budget de l'État.

Par ailleurs et toujours dans cette logique, le gouvernement royal s'est fixé, pour chaque niveau de l'enseignement, des objectifs spécifiques :

- réformer les programmes et les manuels scolaires pour toutes les disciplines et pour toutes les classes;

- augmenter la production et activer la distribution des manuels scolaires;

- améliorer le rendement et l'efficacité de l'enseignement primaire ;

- adapter le système éducatif aux réalités et aux besoins du pays et de son développement.

Le rendement du système éducatif demeure très faible au point que plus de la moitié des enfants sont exclus de l'école avant d'avoir atteint un niveau d'alphabétisation suffisant pour espérer pouvoir le conserver durablement. La proportion de redoublement en première année du primaire est de l'ordre de $42 \%$ et le taux de redoublement dans l'ensemble de ce cycle est en moyenne de $25 \%$. Le nombre d'abandons est également très élevé $(7,4 \%$ en première année et plus de $19 \%$ en cinquième). Le suivi d'une cohorte de 1000 élèves qui entrent en première année de l'enseignement primaire indique que seulement 27 arrivent à la fin du second cycle de l'enseignement général. Cette inefficacité de l'instrument d'éducation traduit avant tout la persistance du manque de qualité de l'enseignement de base, en dépit des efforts 
importants déjà déployés, notamment pour relever le niveau académique et la qualification professionnelle des maîtres.

\section{Organisation générale du système éducatif et démographie scolaire}

16 L'organe exécutif suprême en matière d'éducation est le conseil des ministres, lequel prend, en vertu des lois en vigueur, les arrêtés et dispositions qui régissent le système d'éducation. L'organe central chargé de l'administration du système éducatif, le MEJS, est investi des différentes tâches de conception, d'animation, de contrôle et de gestion dans les domaines de l'éducation, de la jeunesse et des sports. Ses principales missions sont les suivantes :

- la formation initiale et continue des enseignants à tous les niveaux du système ;

- la conception, la planification, l'application et l'évaluation de la politique d'éducation, de la jeunesse et des sports ;

- la gestion prévisionnelle de l'implantation des établissements d'enseignement général et technique, privés ou publics;

- la gestion administrative, pédagogique et technique des structures de l'éducation, de la jeunesse et des sports ;

- la conception des différents types de certification et l'organisation des examens finaux et des concours d'entrée dans l'enseignement ;

- l'élaboration des programmes scolaires, des manuels, des matériels didactiques et des divers documents pédagogiques.

Toutes les villes et provinces disposent une direction de l'éducation, de la jeunesse et des sports et chaque district comprend un bureau de l'éducation, de la jeunesse et des sports. Dans les écoles, un comité directeur et un syndicat (association des enseignants) ont été organisés ainsi que l'association de la jeunesse et l'association des parents.

$\mathrm{Au}$ plan de l'organisation générale, le système éducatif cambodgien est, depuis la rentrée scolaire 1996-1997, officiellement structuré en six années d'études primaires (niveau 1 à 6, avec scolarisation dès l'âge de six ans), suivies de trois années d'enseignement secondaire en collège (années 7, 8 et 9) et enfin trois années en lycée. Ce premier cycle du secondaire au collège est théoriquement obligatoire pour les élèves de douze à quatorze ans. L'enseignement secondaire général du second cycle (lycée) n'est, quant à lui, pas obligatoire. S'inscrivant dans la tendance mondiale d'éducation pour tous, la constitution cambodgienne a ainsi fait des années 1 à 9 de scolarisation le cycle d'éducation de base obligatoire.

L'enseignement préprimaire, facultatif, s'adresse aux enfants âgés de trois à cinq ans. Il est dispensé dans des écoles maternelles et presque exclusivement dans des zones urbaines favorisées.

L'enseignement supérieur et spécialisé comprend quatre degrés de formation qui peuvent durer de deux à cinq ans. Actuellement, le Cambodge dispose de cinq universités publiques, trois instituts spécialisés semi-indépendants et six établissements d'enseignement supérieur privés. Ce secteur est en cours de rénovation. Une loi cadre est en gestation avancée et dès qu'elle sera votée, un projet important de cinq ans financé par un prêt de la Banque mondiale sera mis en œuvre. 
21 La durée officielle de l'année académique est de trente-six semaines, mais de nombreuses dérives sont enregistrées, dues notamment aux conséquences climatiques (inondations) et saisonnières (récoltes).

En ce qui concerne les personnels enseignants, le MEJS a fait des efforts considérables pour former et recycler les maîtres et professeurs aux différents niveaux du système. La hausse du niveau de recrutement des futurs instituteurs du primaire ainsi que l'allongement de la durée de la formation ont été instaurés. Contenus et méthodologie de formation ont été perfectionnés pour garantir un niveau acceptable en termes de compétences, aptitudes et éthique professionnelle.

Quelques indicateurs généraux en éducation ${ }^{1}$

\begin{tabular}{|l|r|}
\hline \multicolumn{1}{|c|}{ Indicateurs } & Valeurs \\
\hline Taux d'alphabétisation moyen des adultes & $65,9 \%$ \\
Taux d'alphabétisation des hommes adultes & $80 \%$ \\
Taux d'alphabétisation des femmes adultes & $53 \%$ \\
Population d'analphabètes de 15 ans et plus & $35 \%$ \\
Taux brut ${ }^{\prime}$ de scolarisation pour les hommes, tous niveaux confondus & $69,5 \%$ \\
Taux brut de scolarisation, pour les femmes, tous niveaux confondus & $54 \%$ \\
Taux brut de scolarisation primaire & $131 \%$ \\
Taux net de scolarisation primaire & $67 \%$ \\
Taux brut de scolarisation secondaire & $24 \%$ \\
Taux net de scolarisation secondaire & $14,1 \%$ \\
Taux brut de scolarisation supérieure & $1,4 \%$ \\
Enfants ne terminant pas le cycle primaire & $50 \%$ \\
\hline
\end{tabular}

1. Sources: Report on the Cambodia Socio Economic Survey, 1997- UNFPA Ministry of Planning, Phnom Penh 1998 ; General population Census of Cambodia, 1998 - UNFPA Ministry of Planning, Phnom Penh 1998 ; Rapport mondial sur le développement humain 1998, PNUD, Annuaire statistique de l'UNESCO, 1998.

2. Le taux brut de scolarisation est le nombre d'élèves inscrits à un cycle (niveau) d'enseignement (qu'ils soient ou non en âge de le suivre), exprimé en pourcentage du nombre d'individus appartenant au groupe d'âge correspondant à ce niveau d'enseignement. Le taux net est le nombre d'élèves inscrits à un cycle (niveau) d'enseignement et qui appartiennent au groupe d'âge pertinent, exprimé en pourcentage du nombre d'individus appartenant à ce même groupe d'âge. Source : Rapport sur le développement humain 1998, PNUD, éd. Economia, 1998.

Actuellement, le Cambodge compte dix-sept écoles provinciales de pédagogie (EPP) ${ }^{1}$ destinées à la formation et au recyclage des enseignants du primaire, six centres régionaux de formation pédagogiques (CRFP) concernant les enseignants du premier cycle du secondaire et une faculté de pédagogie implantée à Phnom Penh et réservée à la formation des professeurs du second cycle et à des activités de recherche.

\section{Financement de l'éducation}

La part de l'éducation dans le plan de développement du gouvernement a augmenté entre 1994 et 1996, passant de 1,5\% à environ 2,1\% du PIB, ce qui est largement dû à l'augmentation des capitaux d'origine externe. En revanche, pendant la période 1997-1998, la part de l'éducation dans les dépenses totales du gouvernement est tombée de $11,8 \%$ à $8,1 \%$, en raison d'une baisse importante du budget national, suite notamment aux douloureux événements de juillet 1997 (combats entre les différentes factions militaires qui ont conduit à l'éviction du premier ministre en place et à la reprise du pouvoir par le parti du peuple cambodgien).

Les dépenses des familles se concentrent sur les cours privés, les livres de classe, les fournitures scolaires, les uniformes, les transports et les repas. Ces contributions 
financières, ajoutées aux honoraires officieux et aux frais de cours privés, constituent un handicap considérable à l'accès aux études des enfants issus des nombreuses couches sociales défavorisées. Le Cambodge détient le peu enviable record régional de la plus faible participation du gouvernement en matière de partage du financement de l'éducation. Selon une étude réalisée par l'UNDP et l'UNESCO, $75 \%$ du coût de l'éducation seraient ainsi imputé aux familles, l'État ne prenant à sa charge que les $25 \%$ restants. À l'exception du Vietnam voisin où le rapport est de l'ordre de $50 / 50$, la tendance est inversée dans tous les pays de la sous-région. Ce constat procure une explication à l'important phénomène d'abandon dont le système scolaire est frappé, particulièrement au niveau de l'enseignement primaire.

La quasi-totalité du budget de l'éducation est absorbée par les dépenses de fonctionnement de l'institution, notamment en matière de versement des salaires aux enseignants et personnels administratifs. Dans ces conditions, on peut considérer que la part du budget réservée aux investissements est, hélas, réduite à sa plus simple expression.

À l'occasion de la publication du plan quinquennal d'investissement 1995-2000, le gouvernement royal s'était engagé à accroître de manière sensible la part du budget de l'État réservée à l'éducation, la faisant passer de $8,76 \%$ à $15 \%$, ce qui devait représenter $2,5 \%$ du produit interne brut. En 2002, l'éducation bénéficierait effectivement de 15 à $17 \%$ du budget de l'État.

\section{Les contraintes du secteur de l'éducation} équipements scolaires, alors que le ratio élèves - maître est en moyenne de 47,2 dans l'enseignement primaire (avec de fortes disparités régionales), que le patrimoine immobilier actuel est en mauvais état (15000 salles de classes sont à rénover dans le primaire) et que plus de 10000 salles de classes s'avèrent nécessaires, au niveau du primaire uniquement, pour espérer éradiquer l'analphabétisme et absorber les effets de la croissance démographique actuelle ;

31 le paupérisme de l'enseignant cambodgien qui, avec environ 25 US\$ de salaire mensuel, n'est pas en mesure de faire vivre sa famille et pratique fréquemment deux à trois métiers. Par voie de conséquence, à de rares exceptions près, les enseignants n'assurent qu'une faible partie de l'horaire officiel d'enseignement. Ils prodiguent en revanche bien souvent un enseignement à mi-temps et multiplient les cours particuliers payants ;

32 le manque d'efficacité de l'enseignant dans sa classe, lié d'une part à des capacités professionnelles limitées, d'autre part à des moyens de communication et d'expression insuffisamment performants ; 
33 la proportion importante de jeunes que le système éducatif ne prend pas en considération et laisse actuellement pour compte, notamment parmi les jeunes filles, les enfants en situation d'échec scolaire, les handicapés et les jeunes des minorités ethniques. Ces différents groupes sociaux représenteraient, ensemble, plus de $50 \%$ de la population en âge d'être scolarisée ;

le caractère formel, général et monolithique de l'enseignement fondamental qui n'offre pas aux enfants devant quitter l'école l'opportunité de développer leur sens pratique et de se familiariser avec un apprentissage de type non cognitif et ne les prépare pas à entrer dans la vie active et à jouer un rôle productif dans la société. Actuellement, l'enseignement technique n'accueille que $1 \%$ environ des élèves, essentiellement du secondaire supérieur. Alors qu'elle constitue un rouage essentiel du développement socio-économique du pays, la formation professionnelle en est au Cambodge à ses balbutiements.

Comme nous pouvons le constater à la lecture de ces derniers points, la tâche majeure à laquelle le MEJS est confronté consiste à assurer une meilleure articulation entre l'enseignement et l'insertion sociale. Dans l'optique d'une ouverture sur la vie et d'une préparation à la profession, l'amélioration de l'éducation et de la formation de base s'inscrivent nécessairement dans un processus progressif et dans une perspective d'éducation permanente, avec la nécessité de prendre en compte les besoins présents et futurs de la société khmère et ceux du citoyen cambodgien aux différentes étapes de son existence.

\section{Pauvreté et éducation}

Le phénomène de pauvreté est endémique au Cambodge. Les estimations fournies par le Cambodia Socio-Economic Survey de 1997 suggèrent que 36 \% de la population vit en dessous du seuil de pauvreté. On constate cependant une certaine amélioration en la matière, puisque les chiffres précédents de 1993/94 stipulaient alors que $39 \%$ de la population vivaient en deçà de ce minimum vital. Mais ce recul théorique de la pauvreté s'avère extrêmement modeste si l'on considère la croissance économique dont le pays a bénéficié durant cette période.

Les raisons de cette relative stagnation de la situation s'expliquent par le fait que la croissance économique a été associée à une augmentation significative des inégalités en matière de consommation entre les différentes strates de la société khmère.

Les données nouvelles collectées à l'occasion du dernier recensement par le ministère du plan, laissent ainsi à penser que la pauvreté s'est légèrement intensifiée dans les campagnes au cours de ces trois dernières années et que les écarts entre les zones urbaines et rurales ont augmenté. Ainsi les ruraux cambodgiens constituent-ils la grande majorité des illettrés et analphabètes. Durant cette même période, la différence entre le nombre d'années de fréquentation scolaire des enfants issus des classes privilégiées et celui issus des enfants des classes défavorisées s'est également accentuée. Une des explications à ce phénomène viendrait de l'élévation du coût de la scolarité qui pénaliserait davantage les enfants des zones rurales, où plus de $80 \%$ des foyers sont gérés par des personnes aux moyens financiers limités et dont le niveau d'éducation dépasse rarement la fin du cycle primaire. L'analphabétisme s'avère ainsi à la fois la cause et le résultat de la pauvreté. 


\section{Les groupes vulnérables et l'éducation}

39 L'une des conséquences directes de la guerre est le nombre aujourd'hui encore important de femmes seules, d'orphelins et de personnes handicapées, suite à des blessures bien souvent provoquées par des mines (on en dénombrerait encore actuellement entre 5 et 7 millions suivant les estimations ${ }^{2}$ ).

Une enquête financée par la Banque Asiatique de Développement (BAD) en 1997 a fourni quelques estimations en matière de composition des groupes les plus défavorisés et des indications relatives à leurs besoins. Ces données évaluent le nombre total de personnes concernées à un demi million. Malheureusement, étant donné l'ampleur des problèmes à résoudre actuellement, ces groupes sociaux ne sont pas ou peu associés au processus de développement et de grandes disparités peuvent ainsi être observées en matière d'accès à l'éducation. De plus, les fragiles institutions cambodgiennes n'apparaissent pas nécessairement préparées à affronter la problématique spécifique de ces groupes particuliers.

41 Malgré tout, dès 1998, le MEJS a lancé un programme national d'alphabétisation dont l'objectif était de réduire de $20 \%$ le nombre d'adultes analphabètes âgés de quinze à quarante-cinq ans. Dans cette perspective, 700 enseignants ont été spécialement formés et plusieurs milliers de livres imprimés.

42 Par ailleurs, plusieurs agences internationales (UNESCO, UNICEF) et ONG se sont impliquées dans le processus global, en partenariat avec des institutions nationales telles que le MEJS, le ministère de l'action sociale ou encore le secrétariat d'État aux anciens combattants ou à la condition féminine.

\section{Évolutions récentes et perspectives du secteur éducatif}

En 2001, le MEJS, suite à une concertation avec les ministères de l'Économie et des Finances, du Plan et de l'Intérieur, avec le Conseil des réformes administratives et les structures administratives décentralisées et en partenariat avec les principaux bailleurs de fonds, a établi une nouvelle série de documents de travail et de perspectives pour le secteur éducatif dont Education strategic plan 2001-5 et Education sector support program 2001-5. Ces productions sont à considérer comme des cadres d'action évolutifs, qui seront évalués annuellement et dont les orientations seront éventuellement précisées ou redéfinies selon les résultats observés.

44 Ces stratégies ont été bâties à partir des travaux qui ont conduit à l'élaboration du "plan stratégique intérimaire de réduction de la pauvreté», du «plan de développement socio-économique 2001-6 », et de l'évaluation nationale du programme " éducation pour tous".

45 Ces plans de travail, profondément ancrés dans la mouvance des concepts fondamentaux de "l'éducation pour tous", doivent contribuer à un élargissement significatif de l'accès à une éducation de qualité, dans une perspective globale de réduction de la pauvreté. Dans ce cadre, les barrières économiques précédemment évoquées et qui pénalisaient les familles les plus pauvres devraient progressivement disparaître. Simultanément, l'accent sera mis sur la qualité, la pertinence et le management général de l'éducation. 
Les processus de décentralisation et de déconcentration précédemment initiés seront renforcés pendant cette période, pour mettre en place une nouvelle architecture des pouvoirs, en conférant aux autorités provinciales, de districts et communales, ainsi qu'aux communautés scolaires, davantage de compétences. Dans cette logique, de nouveaux partenariats devront être initiés, notamment avec le secteur privé et en particulier dans le domaine de la formation professionnelle. Cette vision à long terme a pour objectif d'impliquer au maximum tous les partenaires de l'éducation, afin d'en améliorer la qualité et la pertinence à tous les niveaux du système.

Dans le contexte de ces programmes de décentralisation et de déconcentration, la collecte de données relatives à l'éducation a été développée ainsi que son exploitation aux niveaux provincial et central. Ceci devrait permettre au MEJS, à terme, d'être en possession d'une batterie d'indicateurs fiables, qui autoriseront une meilleure planification du système, une gestion cohérente des ressources humaines et l'élaboration d'une carte scolaire digne de ce nom (qui pourra cependant s'appuyer sur les travaux déjà réalisés à l'occasion de la mise en œuvre du programme CREP construction et réhabilitation des écoles primaires - de la Commission européenne). Un très récent programme spécifique, initié par la $\mathrm{BAD}$, ira dans ce sens en conférant aux structures provinciales les compétences en matière de constructions scolaires.

Enfin, un ambitieux programme quinquennal de réforme de l'enseignement supérieur est en gestation avancée, dans le cadre d'un important prêt de la Banque Mondiale. Une loi cadre devrait être ainsi prochainement votée et la mise en œuvre du projet devrait débuter dans le courant de l'année scolaire 2002/2003. À ce niveau également, la tâche est lourde et complexe. Même si on estime que moins de $2 \%$ d'une classe d'âge atteint ce stade de la scolarité, la demande sociale pour l'enseignement supérieur reste plus élevée que l'offre actuelle. Il s'agit donc de former des diplômés d'université plus compétents et adaptables, en améliorant la pertinence et la qualité de l'enseignement supérieur.

49 Les préoccupations immédiates du secteur tertiaire concernent tout particulièrement les budgets, les équipements, les infrastructures, la gestion administrative et financière, l'accès facilité à l'enseignement supérieur, les procédures d'admissions des étudiants, l'accréditation et l'amélioration de la gouvernance pour pallier les insuffisances actuelles du système.

Il s'agit également de former de nouveaux enseignants et de recycler les plus anciens, en partenariat avec des universités ou instituts techniques étrangers. Les chercheurs de haut niveau et les ingénieurs devront être formés correctement, mais les techniciens supérieurs seront également particulièrement concernés. La mise en place de ce nouveau cadre juridique guidera la croissance et le développement de l'enseignement supérieur en privilégiant simultanément la capacité du Cambodge à gérer les problématiques majeures du développement national, en termes globaux d'éducation, de santé, d'environnement, de gouvernance, de gestion économique et sociale et de développement du secteur privé. 


\section{BIBLIOGRAPHIE}

Annuaire statistique, UNESCO, 1998.

Atlas mondial Microsoft Encarta 2000, Microsoft products.

Cambodge Laos, éditions Arthaud, Paris, 1996.

Cambodia Socio Economic Survey, UNFPA - Ministry of Planning, Phnom Penh, 1997.

Demographic Survey of Cambodia, UNFPA - Ministry of Planning : National Institute of Statistics, Phnom Penh, 1996.

Education strategic plan 2001-5 et Education sector support program 2001-5, gouvernement royal du Cambodge, ministère de l'Éducation, de la Jeunesse et des Sports, Phnom Penh, 2000.

État de l'école (L'), ministère de l'Éducation nationale, de la Recherche et de la Technologie, Paris, octobre 1999.

État du monde (L'), éditions La Découverte, 2000.

GARNIER E. (1995) : Étude sur la participation des familles et des communautés à la vie scolaire et à l'amélioration de l'école au Cambodge, SIPAR, Phnom Penh.

GARNIER E. : Analyse du processus de mise en route et de suivi d'un programme de formation professionnelle d'insertion dans la province de Siem Reap au Cambodge, mémoire de DESS, Paris V, Sorbonne, octobre 2000.

General Population Census of Cambodia, 1998, UNFPA - Ministry of Planning : National Institute of Statistics, Phnom Penh, 1998.

Rapport mondial sur le développement humain, PNUD, 1998.

Rapport mondial sur l'éducation, UNESCO, 2000.

Recensement 1998, résultats provisoires, FNUAP, Phnom Penh, 1998.

RENOU G., GARNIER E. (1999) : Basic Education for all in Cambodia, A strategic approach for human development and poverty alleviation, UNDP - UNESCO, Phnom Penh.

\section{NOTES}

1. EPP : établissements comparables aux anciennes écoles normales en France.

2. Handicap International, Cambodian Mine Action Center, Mine Advisory Group, COFRAS...

\section{INDEX}

Index géographique : Cambodge

Mots-clés : système éducatif, éducation de base 
AUTEURS

\section{ERICK GARNIER}

Chargé de programme au département coopération en éducation du CIEP

GÉRARD RENOU

Senior expert en éducation auprès des grandes agences internationales de développement 